Kompass

Onkologie

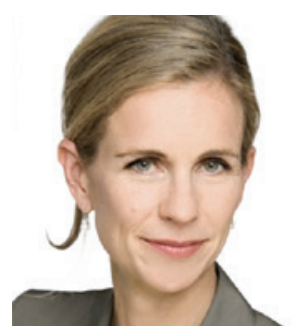

\section{Marion Subklewe}

Klinikum der Universität München, München, Deutschland

\title{
Wir stehen vor einer Dekade der personalisierten T-Zell basierten Therapiestrategien
}

Knapp 3 Jahre sind vergangen, seitdem in Europa die ersten chimären Antigenrezeptor-(CAR)T-Zellen für die Therapie von Patienten mit fortgeschrittenen aggressiven BZell-Lymphomen zugelassen wurden. Die Therapieplattform hat beeindruckende Erfolge in der Behandlung von Patienten mit rezidiviertem und refraktärem diffus großzelligem B-Zell-Lymphom ( $r / r$ DLBCL) und B-Vorläufer akuten lymphatischen Leukämien (BCP-ALL) aufgezeigt. Im Oktober 2020 wurde eine weitere CAR-T-Therapie (Brexucabtagen-Autoleucel) für das $r / r$ Mantelzellymphom durch die EMA zugelassen. Weitere Zulassungen in Europa werden erwartet, die das Spektrum von unterschiedlichen CAR Produkten auf andere Indikationen erweitern werden. Die FDA hat bereits Lisocabtagen-Maraleucel (Liso-Cel) als drittes CAR Produkt bei den aggressiven großzelligen B-Zell-Lymphomen ( $\mathrm{LBCL}$ ), Idecabtagen-Vicleucel (Ide-Cel) bei fortgeschrittenem Multiplen Myelom und Axicabtagen-Ciloleucel (Axi-Cel) beim fortgeschrittenen follikulären Lymphom zugelassen. Klares Zwischenfazit: CAR-T-Zellen sind gekommen, um zu bleiben. CAR-T-ZellTherapien werden in weiteren Indikationen und wahrscheinlich auch in früheren Therapielinien ihren Platz finden.

\section{Was wir aktuell von der CAR-T-Zell- Therapie wissen}

Unsere Erfahrungen im Management von CAR-T assoziierten Nebenwirkungen, in erster Linie Zytokin-Freisetzungssyndrom (cytokine release syndrome, CRS) und Immuneffektorzell-assoziiertes Neurotoxizitätssyndrom (Immune effector Cell-Associated Neurotoxicity Syndrome, ICANS), ist deutlich angewachsen. Wir können diese Therapie in den meisten Fällen ohne höhergradige Toxizitäten sicher umsetzen. Allerdings stehen viele Zentren weiterhin vor logistischen $\mathrm{He}$ rausforderungen. Es bleibt schwierig, den Patienten im bestmöglichen Zustand und unter ausreichender Krankheitskontrolle der CAR-T-Zell-Therapie zuzuführen.

\section{«CAR-T-Zell-Therapien werden in weiteren Indikationen und wahrscheinlich auch in früheren Therapielinien ihren Platz finden.»}

Die Langzeitdaten der Zulassungsstudien von Axi-Cel und Tisagenlecleucel (Tisa-Cel) bei den $r / r$ DLBCL sind beeindruckend und zeigen ein stabiles Plateau. Auch außerhalb von Studien bestätigt sich, dass die Mehrheit der Patienten, die 6 Monate nach CAR-
T-Zell-Therapie eine Remission erreicht haben, diese auch über einen Zeitraum von $>4$ Jahre halten und somit potenziell geheilt sind. Verschiedene US Konsortien konnten bisher überzeugend in großen Patientenkohorten darlegen, dass Patienten, die mehrheitlich nicht die Zulassungskriterien der initialen Studien erfüllten, ein sehr ähnliches Ansprechen sowohl bei progressionsfreiem Überleben (PFS) also auch Gesamtüberleben (OS) aufzeigen. Dieses unterstreicht die Beobachtung, dass nicht die Studieneinschlusskriterien, sondern alternative Parameter, wie metabolisches Tumorvolumen, Laktatdehydrogenase-Wert (LDH-Wert) aber auch Marker der Inflammation von größerer Relevanz für den Therapieerfolg von CAR-T-Zellen sind. Da diese aufkommenden Biomarker leider nicht in allen Studien systematisch erfasst wurden, sind Vergleiche zwischen CAR-T-Zentren oder auch länderübergreifende Vergleiche nur eingeschränkt möglich. Hinzu kommen unterschiedliche Auswertestrategien (PET-CT vs. CT) und -kriterien (Best Response vs. Overall Response) zu unterschiedlichen Zeitpunkten.

An unserem Zentrum konnten wir bisher >60 Patienten mit r/r DLBCL mit CAR-T-Zellen behandeln. Mittels PET-CT evaluierten 
wir eine Gesamtansprechrate von 53\% und eine Komplettremissionsrate von 43\%. Wie u.a. bereits von österreichischen oder französischen Kollegen berichtet, sehen auch wir, dass Patienten mit einem erhöhten LDHWert zum Zeitpunkt der Apherese einen deutlich schlechteren Verlauf haben. Der Erfolg der CAR-T-Zell-Therapie unterliegt auBerdem einem zeitkritischen Faktor. Trotz vorhandener Bridging Konzepte, soll die Systemtherapie aggressiver hämatologischer Neoplasien möglichst bald nach der Erstvorstellung am Zentrum starten. In unserer Patientenkohorte erfolgte bei knapp 50\% der Patienten eine sogenannte «holding» Therapie, also eine Therapie vor Leukapherese. Über 80\% der Patienten erhielten eine sogenannte Bridging Therapie, also eine weitere Therapie vor der eigentlichen Lymphodepletion und anschließender CAR-T-ZellTransfusion. Das bedeutet, dass unsere Patienten eine CAR-T-Zell-Therapie häufig nach bereits 4 Vorlinientherapien erhalten. Die Patienten sind dadurch weiteren Organtoxizitäten ausgesetzt und verschlechtern sich potenziell in ihrem Allgemeinzustand.

\section{Nebenwirkungen der CAR-T-Zell- Therapie}

Insbesondere Hämatotoxizität, die häufigste Nebenwirkung nach CAR-T-Zell-Therapie, mit einer kumulativen 1-Jahres-Inzidenz von 58\% (CTCAE-Grad 23), stellt eine große Herausforderung dar. Die häufig beobachteten Zytopenien sind in knapp der Hälfte der Patienten biphasisch, mit einem erneuten Abfall der neutrophilen Granulozyten und Thrombozyten nach Tag 21 post CAR-T-Zell-Therapie. Die Ursache hierfür ist bisher unzureichend verstanden. Jedoch konnten wir einen prädiktiven Score entwickeln, der die Wahrscheinlichkeit einer schweren Neutropenie (Neutrophile < 500/ul für > 14 Tage) vorhersagt. Dies gelingt durch die Integration von Parametern des Blutbildes ( $\mathrm{Hb}$, ANC, Thrombozyten) und Parametern der Inflammation (CRP, Ferritin) vor Start der Lymphodepletion.

\section{Schlüsselpunkte zum Erfolg der CAR-T-Zell-Therapie}

Um die CAR-T-Zell-Therapie für jeden einzelnen Patienten zum Erfolg zu führen, benötigen wir ein besseres Verständnis für die Schlüsselpunkte im Therapieprozess: Patientenselektion, Leukapherese und CAR-TProduktion, Selektion der geeigneten Bridging Therapie und natürlich ein noch besseres Toxizitätsmanagement. Beim diesjährigen ASH-Kongress erwarten wir die Daten aus den randomisierten Studien in der 2nd Line Therapie bei den LBCL und ich vermute, dass CAR-T-Zellen in der früheren Therapielinie zumindest für bestimmte $\mathrm{Pa}$ tientengruppen eine Zulassung erhalten werden. Mit dem wachsenden Arsenal von Small Molecules und Bispecifics werden wir in Zukunft CAR-T-Zellen auch in Kombination oder früh nach unzureichendem Therapieansprechen zum Einsatz bringen. Die nächste Dekade wird durch personalisierte T-Zell-basierte Therapiestrategien bestimmt werden. 